\title{
Editorische Notiz
}

\section{Ringo Rösener, Felix Bielefeld, Carsten Kinder}

Die Ceschichte von Heinrich Blüchers Vorlesungen an der New School ist mindestens zweigeteilt. Zum einen ist da Heinrich Blüchers Hintergrundgeschichte als deutscher Einwanderer ohne akademische Ausbildung, der die berühmte Denkerin Hannah Arendt heiratete und es auf wundersame Weise an die New School (New York City) und später an das Bard College (Annandale-on-Hudson) schaffte. Diese Geschichte wird ausführlich in der Einleitung dargestellt. Dazu gibt es aber noch die Geschichte, wie seine Vorlesungen ihren Weg in unsere Gegenwart gefunden haben, denn Heinrich Blücher hatte seine Gedanken nirgends zu Papier gebracht. So ist es erstaunlich, dass wir seine Vorlesungen heute lesen können. Wie konnte das geschehen?

Alles begann mit seinen begeisterten Studierenden an der New School. In den 1950er Jahren war die New School in zwei getrennte Bereich aufgeteilt. Der bekannteste Teil war die Graduate Faculty of Political and Social Science, deren Ursprünge auf die University of Exile zurückgingen. In der Graduate Faculty lehrte die ehemalige akademische Elite Europas, die wegen der Nazi-Diktatur fliehen musste. Die Craduate Faculty war eine Art College, an dem Studierende Abschlüsse erwerben konnten, wie an anderen amerikanischen Colleges und Universitäten. Den Kern der New School in den 1950er Jahren bildete jedoch das Adult Education Program, mit dem die New School vor allem Nicht-Akademiker*innen erreichen wollte. Hauptsächlich besuchten New Yorker Bürger*innen, die tagsüber arbeiteten, die Abendkurse des Adult Education Program, um entweder ihr Wissen zu erweitern oder weitere intellektuelle Anregung zu finden. ${ }^{1}$ In diesem Bereich hielt Heinrich Blücher von 1951 bis 1959 fast jeden Freitagabend eine Vorlesung über Philosophie und Kunstphilosophie. Er faszinierte seine Studierenden nicht nur mit der Aura eines europäischen Professors, er betrieb auch eine sehr unkonventionelle Art der Philosophierens. Es dauerte deshalb auch nicht lange bis Blüher bei seinen Zuhörer*innen äußerst beliebt war. Da ihnen klar gewesen sein dürfte, dass Blücher niemals ein Buch über seine Philosophie veröffentlichen würde, begannen seine Studierenden die Vorlesungen auf Tonband aufzunehmen.

Heute werden in der Stevenson Library des Bard College 101 Magnettonbänder mit Aufnahmen von Blüchers Vorlesungen an der New School aufbewahrt. Hannah Arendt selbst übergab sie der letzten Wirkungsstätte ihres Manns, nachdem sie sich vergeblich um die Veröffentlichung der Vorlesungen bemüht hatte. Für dieses geplante Vorhaben hatte sie kurz nach Blüchers Tod 1970 ehemalige Studierende von ihm gebeten, die über

\footnotetext{
${ }^{1}$ Die Ceschichte der New School wird am besten von Judith Friedlander beschrieben: A Light in Dark Times. Die New School for Social Research und ihre Universität im Exil. New York: Columbia University Press 2019.
} 
die Jahre angesammelten Tonbänder zu transkribieren und für eine Edition vorzubereiten. So entstanden erste Transkriptionen und sogar Manuskriptfassungen. Leider haben weder Arendts Bemühungen um eine Publikation noch die Arbeit der Studierenden zu einer Veröffentlichung der Vorlesungen geführt. Arendt gab auf und übergab den größten Teil deshalb an das Bard College. ${ }^{2}$

Im Zuge des Veröffentlichungs-Projektes war Alexander Bazelow mit der Arbeit an den »Sources of Creative Power « beschäftigt. Er arbeitete vor allem schon vorhandene Transkriptionen der Vorlesung in eine neue Manuskript-Fassung um. Die vorhandenen Transkriptionen wurden wahrscheinlich von Ruth Schulz, New School-Zuhörerin und Initiatorin der Aufnahmen, schon in den 1950er Jahren angefertigt. Wir nehmen das vor allem deshalb an, da von der gesamten Vorlesungsreihe zwar fast alle angefertigten Transkriptionen überliefert sind, aber nicht alle Tonbandaufzeichnungen. Es scheint, dass die Tonbänder nach der Erstellung der Transkription wieder mit neuen Vorlesungen Blüchers überspielt wurden. Das würde zumindest erklären, weshalb von den »Sources of Creative Power « nur nicht mal die Hälfte der Ton-Bandaufzeichnungen überliefert, aber fast alle Vorlesungstranskriptionen erhalten geblieben sind.

Bis heute bewahrt das Archiv des Bard College neun Magnettonbänder auf, die als Aufnahmen von »Sources of Creative Power « gekennzeichnet sind. Dank George Rose wurden alle diese Bänder in den 1980er Jahren auf 21 Kassetten (mit einer Laufzeit von jeweils ca. 60 Minuten, insgesamt 42 Stunden) überspielt und in den letzten Jahren digitalisiert. Zusätzlich sind 28 Vorlesungen der Reihe »Sources of Creative Power « transkribiert worden. 24 davon durchliefen einen Bearbeitungsprozess. Vermutlich hat Alexander Bazelow das in den 1970er Jahren getan, um die Vorträge für die von Arendt gewünschte Veröffentlichung vorzubereiten. Aus diesem Grund werden im Bard College drei Arten von Dokumenten dieser Vorlesungsreihe aufbewahrt: 1. die Audioaufnahmen, 2. die Transkription aus den vermutlich 1950er Jahren und 3. Manuskriptfassungen der Vorlesungen aus den 1970er Jahren. Wir veröffentlichen mit dieser Publikation vor allem die Manuskriptfassung.

Was haben wir getan und wie haben wir das gemacht? Zunächst einmal haben wir alle Transkripte und Manuskriptfassungen in der Stevenson Library des Bard College fotografiert. Da die ersten Transkripte oft mühsam zu lesen und für englische Muttersprachler schwer zu verstehen sind, entschieden wir uns, hauptsächlich mit den Manuskriptfassungen zu arbeiten. Was war und ist das Problem mit Blüchers Englisch? Blücher sprach grammatikalisch korrektes Englisch, aber mit einem starken deutschen Akzent, und vor allem übersetzte er deutsche Redewendungen und Sprüche oft wörtlich. Aus diesem Grund sind einige seiner Sätze und Redewendungen für eine Leserschaft, die keinen deutschen Hintergrund hat, oft unverständlich. Dank der Manuskriptfassungen sind die meisten dieser »Fehler« korrigiert worden. Allerdings (sic!) können wir nicht garantieren, dass sich nicht doch noch andere Fehler in diese Manuskriptfassungen eingeschlichen haben oder, noch schlimmer, dass der

\footnotetext{
${ }^{2}$ Der Prozess von Arendts Bemühungen, die Beteiligung der Studierenden und das Scheitern der Veröffentlichung ist in den Hannah Arendt Papers in der Library of Congress dokumentiert. Mappe: Family Papers / Blucher, Heinrich - Writings - Veröffentlichung von Bluchers Manuskripten und Korrespondenz (Box 7, Box 8).
} 
ursprüngliche Cedanke Blüchers verloren gegangen ist. Dennoch sind wir davon überzeugt, dass die Lektüre der Manuskriptfassungen viel Erfolg versprechender ist als der Versuch, die ersten Transkriptionen zu studieren. Einige der Vorlesungen sind jedoch nicht in einer Manuskriptversion überliefert. Hier haben wir stattdessen die ersten Transkripte aus den 1950 er Jahren verwendet, die auf gelben Papier angefertigt wurden. In diesen Fällen haben wir gelb als Farbe der Seiten beibehalten. Was haben wir sonst noch gemacht?

In einem ersten Schritt verbesserten wir die Fotos der Manuskripte und wandelten sie in PDF-Dokumente um (Original Script). In einem zweiten Schritt überprüften wir die Manuskripte und machten daraus komplett neue Manuskriptfassungen (Edited Script). Wir korrigierten einige kleinere Fehler, fügten Quellen und Fußnoten hinzu und setzten lexikalische Links hinter allgemein unbekannte Namen, Begriffe und Philosophen, auf die sich Blücher bezog. Vorzugsweise haben wir uns auf Wikipedia und Wikisource verlassen. Das haben wir absichtlich getan, da wir davon ausgehen, dass Wiki derzeit die langlebigsten Links garantiert und die Informationen ständig auf dem neuesten Stand hält. Wir hoffen , dass alle Links noch lange nach der Veröffentlichung dieser, nun Edited Scripts genannten, Manuskripte funktionieren werden. Diese Edited Scripts können außerdem über OCR (optical character reader) ausgelesen werden. Dort wo wir nur über die Transkriptfassungen verfügten, haben wir ebenso zwei Versionen erstellt, Original Script und Edited Script.

Dennoch haben wir in diesen bearbeiteten Manuskripten (Edited Scripts) auch einige andere Änderungen vorgenommen: Blücher trug seine Vorträge theatralisch vor. Im Cegensatz zu gewöhnlichen Lehrern, die bestimmte Zitate verwenden und versuchen, so sachlich wie möglich zu sein, hielt Bluecher seine Vorträge ganz anders, sozusagen auf eine ganz persönliche Art. Eines dieser Hauptmerkmale ist sein sehr freier Gebrauch von Zitaten und Gedanken der Denker, über die er sprach. Es ist, als brachte er deren Gedanken auf die Bühne des Klassenzimmers der New School. Wie hat er das gemacht? Zunächst nahm er selbst die Position des Denkers ein. Er spielte Lao-tze oder Heraklit. Er zitierte sie und sprach sein Publikum in einer Weise an, von der er überzeugt war, so hätten sie es selbst getan. Zweitens: Er versetzte sich selbst in einen Dialog. Das heißt, er benutzte Fragen wie »Was sollen wir tun?«, »Was können wir tun?«, »Was soll das bedeuten?«, um eine Art fragenden Dialog zu führen. Drittens hob oder senkte er seine Stimme wie ein Ceschichtenerzähler, der ein Abenteuer nacherzählt. Blücher war kein gewöhnlicher Mann der schlicht Philosophie lehrte, er war ein Performer.

Aus diesem Grund hatten wir einige Probleme, echte Zitate von Blüchers eigenen Versionen zu trennen und Blüchers theatralische Darbietungen in lesbare Texte umzuwandeln. Unsere Lösung war, alle echten Zitate, die wir finden konnten, in doppelte Anführungszeichen (» «) zu setzen. Blüchers Versuche, zu rezitieren, einen Dialog zu imitieren oder einen Dialog mit dem Publikum zu führen, haben wir in einfache Anführungszeichen ( $>$ ) gesetzt. Wann immer wir zudem die Originalquelle ausfindig machen konnten, haben wir sie in eine Fußnote gesetzt. Wir hoffen, dass wir Blüchers »Kunst«, Vorträge zu halten, in den Text übersetzen konnten, ohne unnötige Verwirrung zu stiften und ohne seine Originalität zu zerstören.

Bei unseren Recherchen sind wir auf zwei fehlende Vorlesungen gestoßen, die nur auf 
den Magnettonbändern bzw. auf den mit 16b bis 18b gekennzeichneten Kassettenbändern erhalten geblieben sind. Es handelt sich dabei um die Vorlesungen IX und X des Frühjahrssemesters 1954. Diese Fund ist sehr erfreulich, da diese Vorlesungen Blüchers Vorträge über Heraklit und Solon enthalten, die im Lehrplan des New School Bulletin angekündigt, aber bisher nicht transkribiert worden sind. Wir haben komplett neue Transkripte angefertigt und sie den Edited Scripts hinzugefügt. Damit ist die gesamte Vorlesungsreihe mit 29 Vorträgen nun komplett.

All dies wäre ohne die Hilfe der Mitarbeiter*innen der Stevenson Library am Bard College nicht möglich gewesen. Daher möchten wir uns bei Helene Tieger und Betsy Cawley bedanken, die beide für das Blücher-Archiv zuständig sind. Ohne ihre unterstützende Hilfe hätte dieses Projekt nicht realisiert werden können. Darüber hinaus gibt es viele weitere Personen aus verschiedenen Jahrzehnten, die dies möglich gemacht haben. Ruth Schulz, Jack Blum und Alexander Bazelow, die sich um den geistigen Nachlass Blüchers kümmerten; George Rose, der die verbliebenen Bänder der »Sources of Creative « Power auf Kassetten überspielte; und nicht zuletzt Jeff Katz, der die ersten Vorträge online stellte (www.bard.edu/bluecher) und damit den ersten Schritt machte, Blücher neu zu entdecken. Ein großer Dank geht an Carsten Kinder, der in endlosen Nachtschichten an den Original Skripts und den Edited Skripts gearbeitet hat, an Tom Quasthoff und Jakob Schober, die bei den englischen Texten (Introduction and Editor's) geholfen haben, und an Felix Bielefeld, der die fehlenden Vorträge gefunden und beachtlich viel dazu beigetragen hat, diese Vorlesungsreihe überhaupt zu veröffentlichen. 